\title{
Artery of Percheron Infarction
}

\author{
K.V. Vinod ${ }^{a}$ R. Kaaviya ${ }^{a}$ Bhaumik Arpita $^{b}$ \\ Departments of ${ }^{\mathrm{a}}$ General Medicine and ${ }^{\mathrm{b}}$ Radiodiagnosis, JIPMER, Puducherry, India
}

\author{
Key Words \\ Thalamic infarct · Brainstem stroke · Hypersomnolence · \\ Vertical gaze palsy
}

\begin{abstract}
Artery of Percheron (AOP) occlusion is a rare cause of ischemic stroke characterized by bilateral paramedian thalamic infarcts, with or without mesencephalic infarction. Clinically it presents with mental state disturbances, hypersomnolence, aphasia/dysarthria, amnesia and ocular movement disorders, including vertical gaze palsy. Here, we report a case of cardioembolic AOP infarction in a 37-year-old woman with rheumatic mitral valvular stenosis. This case is being reported to highlight the interesting clinical and neuroimaging features of this rare condition, and the differential diagnosis of AOP infarction on imaging have been discussed.
\end{abstract}

๑) 2016 S. Karger AG, Basel

\section{Introduction}

The thalamus on either side is supplied predominantly by multiple small branches arising from ipsilateral posterior communicating artery and $\mathrm{P}_{1}$ and $\mathrm{P}_{2}$ segments of posterior cerebral artery. Percheron, a French neurologist, was the first to describe possible variations involving the paramedian thalamic-mesencephalic arterial supply in 1976. Artery of Percheron (AOP) is an uncommon an- atomic variant, in which a single, unpaired thalamoperforating artery trunk arises from the first part of the posterior cerebral artery and supplies bilateral medial thala$\mathrm{mi}$, with variable contribution to the rostral midbrain [1]. Occlusion of AOP causes a characteristic pattern of ischemia involving paramedian thalami and rostral midbrain.

\section{Case Report}

A 37-year-old woman was rushed to emergency department in a stuporous state, after she was found unresponsive in the morning. She had no significant past medical problems and addictions. There was no history of fever, headache and vomiting and she was apparently fine on the previous night. Examination revealed an afebrile, deeply drowsy, confused and aphasic lady (Glasgow Coma Scale score: $8 / 15$ ), with regular heart rate of $84 / \mathrm{min}$, blood pressure of $130 / 90 \mathrm{~mm} \mathrm{Hg}$ and respiratory rate of $12 / \mathrm{min}$. She had bilateral ptosis and external ophthalmoplegia. Pupils were semidilated $(6 \mathrm{~mm})$, with sluggish reaction to light and there was no weakness of limbs. Plantar responses were flexor on both sides. Optic fundi were normal. Cardiovascular and other systemic examination was unremarkable. Biochemical laboratory tests and blood counts were normal. Electrocardiogram was within normal limits. Initial CT of the brain at admission was unremarkable. MRI of the brain done later revealed hyperintensities in bilateral paramedian thala$\mathrm{mi}$ and rostral midbrain in $\mathrm{T}_{2}$-weighted images (fig. 1-3), and diffusion-weighted MRI (fig. 4) showed restricted diffusion in the same areas, suggesting acute infarcts. MR cerebral angiography and venography were unremarkable.

She continued to have hypersomnolence during the first week of hospital stay. A diagnosis of AOP infarction was made, based on typical imaging findings and clinical features such as bilateral

\section{KARGER}

E-Mail karger@karger.com

www.karger.com/aon (c) 2016 S. Karger AG, Base

0972-7531/16/0232-0124\$39.50/0
Dr. K.V. Vinod

Department of General Medicine

JIPMER, Dhanvantrinagar

Puducherry 605006 (India)

E-Maildrkvv@ rediffmail 
Fig. 1. Axial $\mathrm{T}_{2}$-weighted MR FLAIR image of the brain showing bilateral paramedian thalamic hyperintensities (arrows), suggesting acute infarcts.

Fig. 2. $\mathrm{T}_{2}$-FLAIR axial image of brain showing rostral midbrain infarct and a $\mathrm{V}$-shaped hyperintense signal intensity along the pial surface of the midbrain at the interpeduncular fossa (the ' $\mathrm{V}$ ' sign, indicated by arrow).

Fig. 3. Coronal $\mathrm{T}_{2}$-weighted $\mathrm{MR}$ image of brain showing hyperintensities in bilateral paramedian thalami (arrows) and rostral midbrain (arrowhead), suggesting acute infarcts.

Fig. 4. Axial diffusion-weighted MR image of brain, showing restricted diffusion (hyperintense signals indicated by arrows) in bilateral paramedian thalami, suggesting acute infarcts.
1

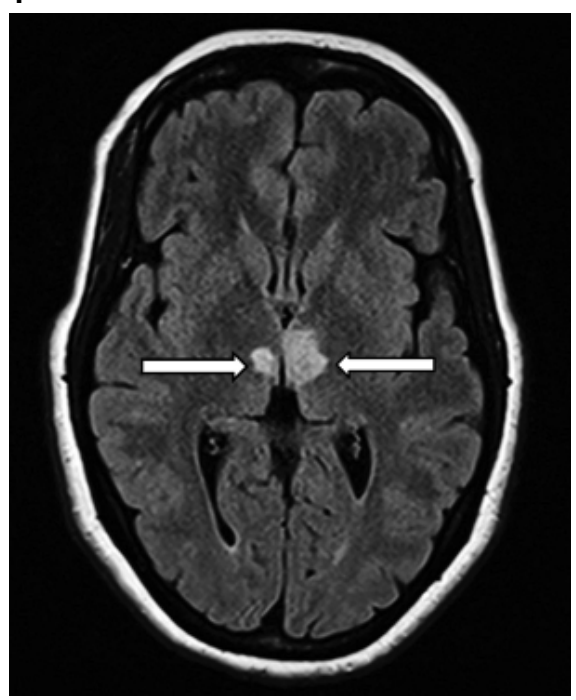

2
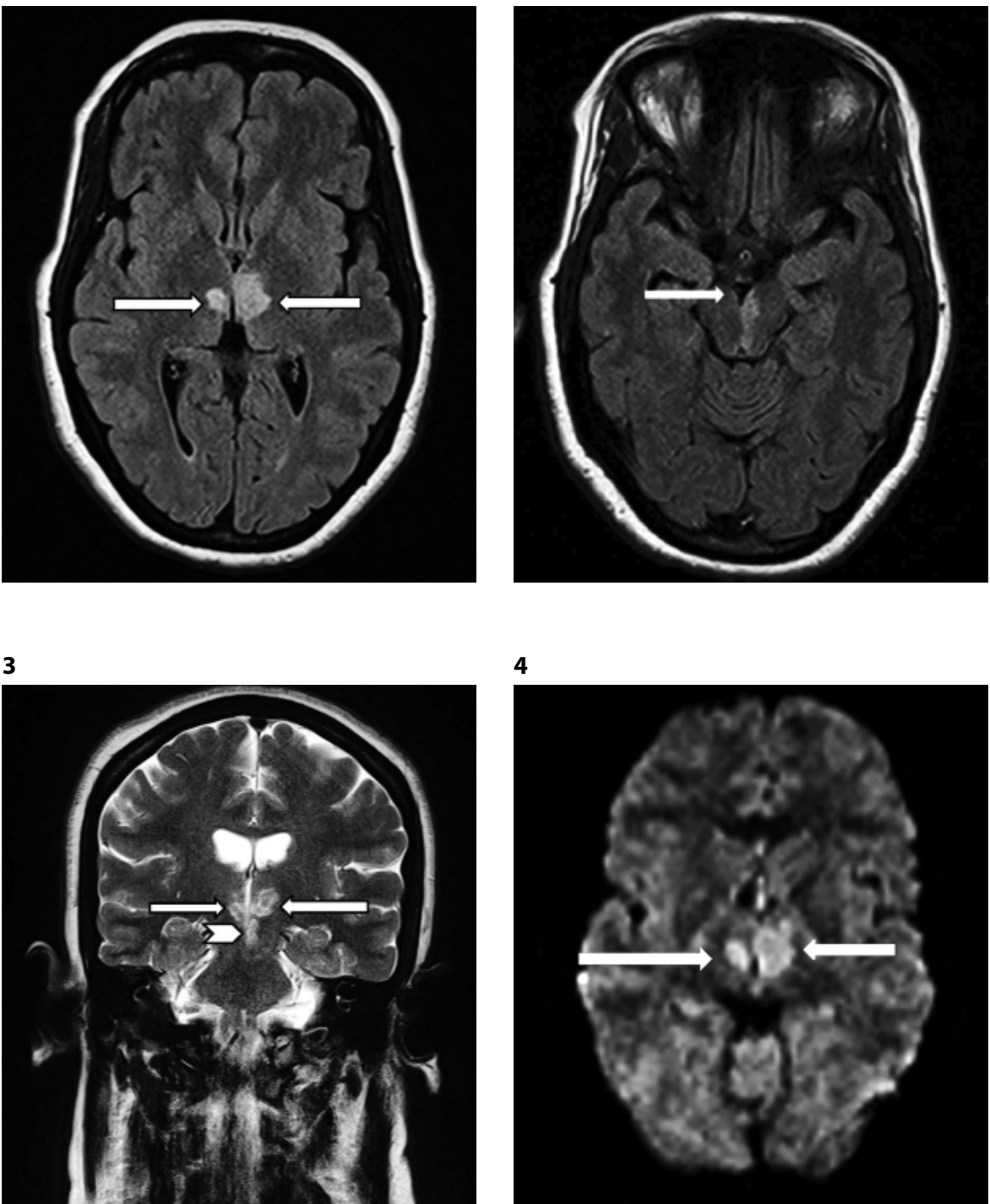

4

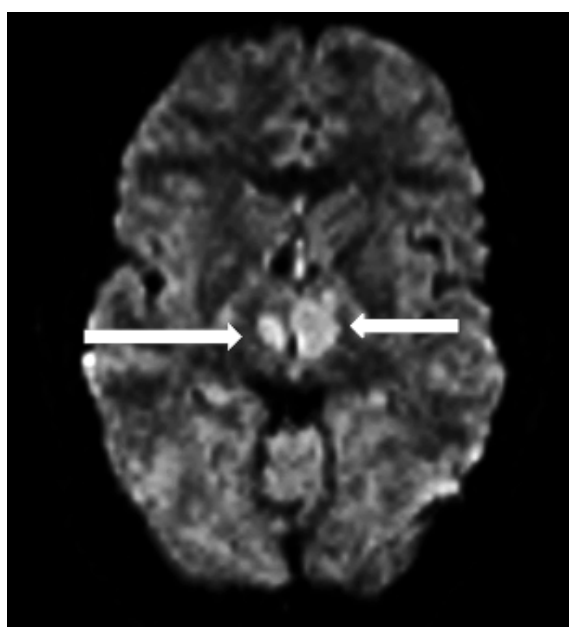

external ophthalmoplegia, alteration in level of consciousness, aphasia and amnesia. Echocardiographic evaluation of the heart revealed rheumatic mitral valvular stenosis of moderate severity. She was started on warfarin for prevention of furthercardioembolic events. She showed gradual improvement ofhypersomnolence, aphasia, ptosis and ocular movements on right side over 2 weeks, but left-sided third nerve palsy has persisted at 1 month follow-up.

\section{Discussion}

Bilateral thalamic infarctions are rare and accounted for $0.6 \%$ of cases of ischemic stroke in a registry documenting 2,750 patients of ischemic stroke [2]. Occlusion of AOP, which results from cardioembolism or arterio- arterial embolism or atherothrombosis, is a rare cause of ischemic stroke and gives rise to characteristic pattern of bilateral paramedian thalamic infarcts, with or without rostral midbrain infarction [3]. Clinical features can be quite variable and include mental state disturbances/ coma, hypersomnolence, aphasia/dysarthria, amnesia, ocular movement disorders (including vertical gaze palsy) and pupillary abnormalities in different combinations [4]. The index patient had confusion, hypersomnolence, amnesia, aphasia, ptosis, complete external ophthalmoplegia and pupillary dilatation on both sides. The $\mathrm{V}$ sign (axial $\mathrm{T}_{2}$ fluid-attenuated inversion recovery (FLAIR) MRI through the midbrain showing a V-shaped hyperintense signal intensity along the pial surface of the midbrain at the interpeduncular fossa), as seen in the 
index case, has been reported as a characteristic neuroimaging finding in AOP infarction [1]. AOP is rarely visualized, even with conventional angiography $[1,5]$ and in the index case MR angiography could not demonstrate it.

It is important for general/emergency care physicians and neurologists to be aware of AOP infarction to properly diagnose and to institute appropriate and timely treatment for this ischemic stroke syndrome. This will avoid further unnecessary investigations and treatments. Successful intra-arterial thrombolysis has been reported in a case of AOP infarction [5]. The 'top of the basilar artery syndrome', which usually results from embolic occlusion of distal basilar artery, closely mimics AOP infarction [6] both clinically and radiologically, as it gives rise to bilateral thalamic infarctions. The 2 posterior cerebral arteries, which are terminal branches of basilar artery supply posterior thalami, geniculate bodies and cerebral peduncles. Distal basilar artery also gives rise to superior cerebellar arteries and pontine perforator branches. Thus, in distal basilar occlusion, apart from bilateral thalamic infarctions, infarction of posterior cerebellar artery, superior cerebellar artery and pontine territories are seen in addition [6]. In addition, MR angiography demonstrates a filling defect in the distal basilar artery. In the index case, MR angiography revealed normal basilar and posterior cerebral arteries.
Other imaging differential diagnoses for AOP infarction, producing bilateral thalamic lesions include deep cerebral venous thrombosis, Wernicke's encephalopathy, extrapontine myelinolysis, viral or demyelinating encephalitis, Wilson's disease, other metabolic and toxic processes, bilateral thalamic glioma and Creutzfeldt-Jakob disease $[6,7]$. These were excluded in the index case based on clinical features and other imaging findings. Long-term clinical outcome in patients of AOP infarction is generally favourable, except in those with midbrain infarction [4]. There was significant recovery in the index case at 4 weeks, barring some residual third nerve weakness on the left side.

To conclude, AOP occlusion is a rare cause of ischemic stroke involving bilateral paramedian thalami. Awareness of the clinical and neuroimaging features of this stroke syndrome is essential for timely diagnosis and appropriate management.

Informed verbal consent has been obtained from patient for publication of her case report and neuroimages and her personal identity has not been revealed anywhere in the manuscript.

\section{Acknowledgment}

None.

\section{References}

1 Lazzaro NA, Wright B, Castillo M, et al: Artery of Percheron infarction: imaging patterns and clinical spectrum. AJNR Am J Neuroradiol 2010;31:1283-1289.

2 Kumral E, Evyapan D, Balkir K, et al: Bilateral thalamic infarction. Clinical, etiological and MRI correlates. Acta Neurol Scand 2001; 103:35-42.
3 Matheus MG, Castillo M: Imaging of acute bilateral paramedian thalamic and mesencephalic infarcts. AJNR Am J Neuroradiol 2003; 24:2005-2008.

4 Arauz A, Patiño-Rodríguez HM, VargasGonzález JC, et al: Clinical spectrum of artery of Percheron infarct: clinical-radiological correlations. J Stroke Cerebrovasc Dis 2014; 23:1083-1088.

5 Kostanian V, Cramer SC: Artery of Percheron thrombolysis. AJNR Am J Neuroradiol 2007; 28:870-871.
6 Rodriguez EG, Lee JA: Bilateral thalamic infarcts due to occlusion of the artery of Percheron and discussion of the differential diagnosis of bilateral thalamic lesions. J Radiol Case Rep 2013;7:7-14.

7 Chatterjee S, Thomas B, Kesavadas C, et al: Susceptibility-weighted imaging in differentiating bilateral medial thalamic venous and arterial infarcts. Neurol India 2010;58:615-617. 